\title{
Funciones ejecutivas y práctica de ajedrez: un estudio en niños escolarizados
}

\section{(1)Psicogente ISSN 0124-0137 EISNN 2027-212X}

\section{Executive functions and playing chess practice: a study conducted with schoolchildren}

\author{
Larisa Ramos \\ Universidad Adventista del Plata, Provincia de Entre Rios, Argentina \\ Vanessa Arán Filippetti Gabriela Krumm \\ CONICET y Universidad Adventista del Plata, Provincia de Entre Rios, Argentina
}

\begin{abstract}
Resumen
Objetivo: Diversas investigaciones han demostrado los beneficios del ajedrez para el desarrollo cognitivo. Sin embargo, son escasos los estudios que han analizado el efecto del ajedrez en las Funciones Ejecutivas (FE) con base en un modelo que evalúe cada uno de los componentes del constructo, el fin del presente estudio fue analizar las diferencias de rendimiento cognitivo en tareas que valoran las FE
\end{abstract} de memoria de trabajo, inhibición, flexibilidad cognitiva y planificación entre niños practicantes de ajedrez y no practicantes

Método: A través de un estudio de tipo ex post facto de corte transversal, con una muestra seleccionada a través de un muestreo no probabilístico intencional, empleando el Análisis Multivariado de Varianza (MANOVA) para analizar las diferencias de rendimiento cognitivo según la práctica de ajedrez. Se evaluaron 65 niños escolarizados de 8 a 12 años, de ambos sexos divididos en dos grupos de acuerdo a la práctica de ajedrez: (1) 30 niños practicantes activos y (2) 35 niños sin ninguna experiencia con el ajedrez

Resultados: El MANOVA reveló diferencias significativas según la práctica de ajedrez, en todas las FE analizadas a favor de los niños practicantes. Estos datos sugieren que el ajedrez podría emplearse como una estrategia efectiva para favorecer el desarrollo de los procesos ejecutivos en la niñez. Se discuten los resultados en función de sus implicancias clínicas y educativas

Conclusiones: Para la realización de futuras investigaciones sería relevante estudiar el efecto de la práctica de ajedrez en las FE en poblaciones adolescentes y adultas, para comprobar si la experticia y la edad podrían explicar, en parte, las variaciones individuales de funcionamiento ejecutivo según la práctica de ajedrez.

Palabras Claves:

Ajedrez, Funciones Ejecutivas, Niños escolarizados, Desarrollo cognitivo.

\section{Abstract}

Objective: Research has demonstrated the benefits of chess for cognitive development. However, few studies have analyzed the effect of chess on the Executive Functions (FE) based on a model that evaluates each of the components of the construct, the purpose of this study was to analyze the differences in cognitive performance in tasks which assess work memory, inhibition, cognitive flexibility, and planning between child chess practitioners and non-practitioners

Method: Through an ex post facto cross-sectional study, using a sample selected through an intentional non-probabilistic sampling, using the Multivariate Analysis of Variance (MANOVA) to analyze differences in cognitive performance according to the practice of Chess. Sixtyfive schoolchildren aged 8 to 12 years old, of both sexes were divided into two groups according to the chess practice: (1) 30 active child practitioners and (2) 35 children with no experience with chess.

Results: MANOVA revealed significant differences according to chess practice, in all FE analyzed in favor of practicing children. These data suggest that chess could be used as an effective strategy to favor the development of executive processes in childhood. The results are discussed according to their clinical and educational implications

Conclusion: In order to carry out future research, it would be relevant to study the effect of chess practice on EFs in adolescent and adult populations, in order to verify if the experience and age could explain, in part, the individual variations of executive functioning according to practice of chess.

Key words: Chess, Executive Functions, Schoolchildren, Cognitive development

Como citar este artículo (APA):

Ramos, L., Arán, V. \& Krumm, G. (2018). Funciones ejecutivas y práctica de ajedrez: un estudio en niños escolarizados. Psicogente, 21(39), 25-34. http://doi.org/10.17081/psico.21.39.2794 


\section{INTRODUCCIÓN}

El deporte es un instrumento de socialización ampliamente utilizado por educadores para promover un desarrollo integral de los estudiantes (Fraile-Aranda, 2004). Para Tamorri (2004), el deporte es un fin en sí mismo y una herramienta que contribuye a mejorar las habilidades mentales del individuo.

Entre los deportes que requieren de habilidades cognitivas de alto orden y tienen efectos positivos sobre el desarrollo cognitivo se encuentra el ajedrez. El ajedrez es una ciencia, un arte, un deporte y una gran herramienta educativa (Fernández-Amigo \& Sánchez-Rincón, 2011). Para Gobet y Campitelli (2006), ser competente en ajedrez requiere la adquisición de un conocimiento especializado, incluyendo la memorización de un gran número de patrones específicos del juego que pueden implicar movimientos apropiados, evaluaciones o planes. Requiere, además, la capacidad de hacer una búsqueda efectiva y evaluar correctamente las posibles posiciones de ajedrez.

Los procesos que se ponen en juego durante la práctica son considerados habilidades transferibles que cumplen un papel importante en el establecimiento y el alcance de metas y en el logro de un aprendizaje auto-regulado (Hong \& Bart, 2007). Diversas investigaciones han demostrado que el ajedrez tiene un impacto positivo en la concentración, la visualización, la previsión, la toma de decisiones, el pensamiento abstracto, la planificación, la memoria (Kovacic, 2012) y el rendimiento académico (Kazemi, Yektayar, \& Abad, 2012; Kovacic, 2012; Thompson, 2003).

Se ha indicado, además, que mejora procesos cognitivos como el pensamiento estratégico, la resolución de problemas (Dauvergne, 2000; Ferreira \& Palhares, 2008; Kazemi et al., 2012) y la inteligencia (Aciego, García \& Betancort, 2012; Gobet \& Campitelli, 2002), entre otros. En los últimos años, estudios neuropsicológicos han comenzado a enfatizar los beneficios de la práctica de ajedrez en las funciones ejecutivas (FE).

Estos procesos cognitivos de alto orden posibilitan la adaptación a situaciones complejas o novedosas, que no son rutinarias y requieren de mecanismos de control para ser resueltas de un modo eficaz (Collette, Hogge, Salmon, \& Van der Linden, 2006; Calle, 2017). Se considera un constructo multidimensional que incluye los subprocesos de:

1) Memoria de trabajo, 2) inhibición y 3) flexibilidad cognitiva (Diamond, 2016).

La memoria de trabajo es el sistema cerebral que posibilita almacenar temporariamente y mantener en la mente la información necesaria para la ejecución de tareas cognitivas complejas (Baddeley, 1992). La inhibición se refiere a la habilidad para suprimir, intencionadamente, respuestas dominantes, prepotentes o automáticas, cuando es necesario (Miyake, Friedman, Emerson, Witzki, Howerter \& Wager, 2000).

Por último, la flexibilidad cognitiva, se define como la capacidad para cambiar o alternar entre diferentes sets mentales o como la habilidad para ver algo desde diferentes perspectivas (Diamond, 
2016). Esta estructura de tres dimensiones o componentes ejecutivos ha sido demostrada tanto en niños de habla finlandesa (Lehto, Juujärvi, Kooistra \& Pulkkinen, 2003) como de habla hispana (Arán Filippetti, 2013). Finalmente, una FE más avanzada que se construye a partir de las tres dimensiones centrales, es la capacidad de planificación (Diamond, 2016; Miyake et al., 2000).

Esta FE implica la posibilidad de plantearse un objetivo, ensayar mentalmente y emplear una estrategia para alcanzarlo y, finalmente, valorar si se logró o no el objetivo planeado (TirapuUstárroz, Muñoz-Céspedes, Pelegrín-Valero \& Albéniz-Ferreras, 2005). En general, los estudios que han analizado los efectos de la práctica de ajedrez en las FE se han centrado en alguno de sus componentes específicos. Así, por ejemplo, Unterrainer, Kaller, Halsband y Rahm (2006) valoraron las dimensiones memoria de trabajo y planificación (mediante la tarea Torre de Londres) en jugadores de ajedrez y no practicantes y hallaron que, los jugadores de ajedrez, presentan un mejor rendimiento en la tarea de planificación. Sin embargo, no hallaron diferencias significativas entre los dos grupos en cuanto a la inteligencia y la memoria de trabajo verbal y viso-espacial.

Por su parte, Cuéllar y Díaz (2009) no encontraron diferencias entre ajedrecistas profesionales, aficionados y no practicantes en el desempeño en pruebas ejecutivas que valoran la capacidad de planificación (Torre de Hanoi, Laberintos de Porteus y el Mapa del zoológico) aunque hallaron que, los sujetos no practicantes de ajedrez, reaccionan más rápidamente para iniciar las actividades y emplean más tiempo para finalizar las tareas, respecto de los practicantes de ajedrez profesionales y aficionados. Según los autores, estos hallazgos sugieren que los sujetos no practicantes de ajedrez presentan una menor capacidad de planificación y un menor autocontrol que los sujetos practicantes.

En cuanto a las dimensiones flexibilidad cognitiva e inhibición, Nejati y Nejati (2012) no encontraron diferencias significativas entre adultos jóvenes practicantes de ajedrez y no practicantes. Finalmente, si bien se ha demostrado el papel del componente visuoespacial y ejecutivo central de la memoria de trabajo en la práctica de ajedrez (Robbins et al., 1996), hay autores que no han hallado relaciones significativas entre la habilidad para el ajedrez y la memoria visual (Waters, Gobet \& Leyden, 2002; Spiendler, Carlotto, Ogliari y Giordani, 2015). Si bien los estudios referenciados hasta el momento se han realizado en poblaciones adultas, existen también investigaciones realizadas con poblaciones infantiles y adolescentes que han demostrado los beneficios del ajedrez para el desarrollo cognitivo.

Así, por ejemplo, Aciego et al. (2012) hallaron que niños y adolescentes practicantes de ajedrez presentan una mejor capacidad de planificación, memoria y mejores habilidades visuoespaciales, en comparación con niños y adolescentes que practican fútbol o baloncesto. Además, los participantes del estudio que jugaban ajedrez presentaron una mejor capacidad de resolución de problemas, afrontamiento y desarrollo socio-emocional. Con relación a la memoria, tanto Horgan y Morgan (1990), como Schneider, Gruber, Gold y Opwis (1993), han encontrado efectos del ajedrez en diferentes tareas que implican la capacidad mnésica. Finalmente, también se han demostrado los efectos del ajedrez en la flexibilidad cognitiva. 


\section{Larisa Ramos, Vanessa Arán Filippetti y Gabriela Krumm}

Por ejemplo, Rojas-Vidaurreta (2001) encontró un mejor desempeño en esta FE, valorada mediante el test de clasificación de tarjetas de Wisconsin en su versión computarizada, en niños de 7 a 11 años practicantes de ajedrez en comparación con niños que no practicaban el deporte. En conjunto, se evidencian algunos estudios que han analizado los efectos del ajedrez sobre algunas FE específicas con diferencias según la edad analizada y la tarea de FE empleada. Sin embargo, dada la escasez de estudios que analicen la relación entre la práctica de ajedrez y la FE en niños de habla hispana, considerando bajo este análisis a las diferentes dimensiones del constructo según uno de los modelos actuales de FE (Miyake et al., 2000; Diamond, 2016), el objetivo del presente estudio fue analizar las diferencias de rendimiento cognitivo en tareas que valoran las FE de memoria de trabajo, inhibición, flexibilidad cognitiva y planificación entre niños practicantes de ajedrez y no practicantes.

\section{MÉTODO}

\subsection{Diseño}

La investigación realizada se corresponde a un estudio de tipo ex post facto de corte transversal (Arnau, 1978; Cortada de Kohan, Macbeth \& López-Alonso, 2008; Montero \& León, 2007), dado que la variable independiente (practicantes de ajedrez vs no practicantes) no es posible de manipular.

\subsection{Participantes}

La muestra, obtenida a través de un muestreo no probabilístico intencional, estuvo conformada por 65 niños, de los cuales $42(64,6 \%)$ eran varones y $23(35,4 \%)$ eran mujeres. El $29,2 \%(n=19)$ de los niños tenía 8 años, el $20 \%(n=13)$ tenía 9 años, el 36,9\% $(n=24)$ tenía 10 años, el 6,2 \% ( $n=4)$ tenía 11 años y el 7,7 \% ( $n=5)$ tenía 12 años.

Del total de participantes, 35 no tenían ninguna experiencia con el ajedrez y asistían a dos instituciones educativas y los otros 30 eran practicantes activos de ajedrez. Los criterios de inclusión para los practicantes de ajedrez fueron:

1) que asistieran a un taller organizado específicamente para niños de su edad, ya sea municipal o privado

2) que llevaran un mínimo de dos años practicando sistemáticamente el deporte.

Para la muestra total se consideró, además, que los niños no presentaran antecedentes clínicos, neurológicos ni psiquiátricos, y que cursasen sus estudios escolares con regularidad, sin repitencia escolar. En cuanto a los niños que eran practicantes activos de ajedrez, la media de edad fue de 9,40 $(D E=1,50)$, siendo 2 niñas y 28 niños, siendo la media del nivel educativo de los padres 7,73 ( $D E=$ $1,14)$, correspondiendo a un nivel universitario incompleto. En relación al grupo de niños que no tenían experiencia, la media de edad fue de 9,29 ( $D E=0,86)$.

El grupo quedó conformado por 21 niñas y 14 niños. En este caso, la media del nivel educativo de los padres fue de 7,83 ( $D E=1,25$ ), correspondiéndose también a un nivel universitario incompleto. En la Tabla 1 se presentan las características sociodemográficas para cada grupo (practicantes de ajedrez vs. no practicantes). 
Dado que la edad es un factor asociado al desempeño en tareas que valoran las FE (Arán-Filippetti, 2011), para controlar esta variable, se realizó una prueba t para muestras independientes para estudiar si existían diferencias significativas en cuanto a la edad según la práctica de ajedrez. Los resultados indicaron que no existen diferencias entre los grupos Practicantes ajedrez vs. No practicantes $(t) 63)=1.014, p=.316)($ ver Tabla 1$)$.

Para acceder a las escuelas y a los talleres de ajedrez, se contactó al personal directivo de las instituciones, explicando los objetivos de la investigación y la modalidad de aplicación de los instrumentos. Teniendo en cuenta que los participantes del estudio eran menores de 18 años, se les entregó un consentimiento para brindar información a los padres o tutores responsables del menor, aclarando que la participación era voluntaria y anónima.

Tabla 1.

Características sociodemográficas de la muestra.

Grupo

\begin{tabular}{lcc}
\hline & Practicantes de ajedrez & No practicantes \\
Número de sujetos & 30 & 35 \\
Edad & $9.60(1.50)$ & $9.29(0.86)^{\mathrm{ns}}$ \\
Sexo & $2 \mathrm{~F} / 28 \mathrm{M}$ & $21 \mathrm{~F} / 14 \mathrm{M}$ \\
Nivel educativo padres $\ddagger$ & $7.73(1.14)$ & $7.83(1.25)$ \\
\hline
\end{tabular}

ns = diferencias no significativas. $¥$ Para el Nivel educativo de los padres se tuvo en cuenta en nivel más alto alcanzado por el padre o madre de cada niño. Las categorías empleadas fueron las siguientes: $0=$ No asistió a la escuela, 1=Escuela primaria incompleta, 2=Escuela primaria completa, $3=$ Secundaria incompleta, $4=$ Secundaria incompleta, $5=$ Terciario incompleto, $6=$ Terciario completo, $7=$ Universidad incompleta, $8=$ Universidad completa, 9=Doctorado en curso, $10=$ Doctorado completo

\subsection{Instrumentos}

2.3.1 Test de Colores y Palabras de Stroop (Golden, 2007) Brinda una medida de la resistencia a la interferencia y la capacidad de inhibición de respuestas verbales. Está integrado por tres láminas. En la primera lámina, se solicita la lectura en voz alta, lo más rápido posible, de las palabras rojo, verde, azul, impresas en mayúscula negra en orden aleatorio. En la segunda lámina, compuesta por grupos de cuatro XXXX impresos al azar en color rojo, verde o azul, se solicita que se nombre el color de la tinta, lo más rápido posible. La última lámina, está constituida por los nombres de los colores de la lámina 1 pero coloreados en rojo, verde o azul de un modo en que no coincida el nombre con el color. En esta última instancia, se le solicita al niño que mencione, lo más rápido posible, el color de la tinta. La puntuación directa obtenida en la lámina palabra-color, fue la utilizada como medida de inhibición y capacidad de interferencia, en tanto demanda inhibir la lectura de la palabra para denominar el color de la tinta. 


\subsubsection{Memoria de trabajo, WISC IV (Wechsler, 2010)}

Está compuesto por dos subtest principales:

2.3.2.1. Dígitos (D): consiste en la lectura por parte del examinador de una serie de dígitos, en complejidad creciente, y de su repetición por parte del niño. Este subtest ofrece una medida de la retención verbal inmediata cuando se evalúa con dígitos en orden directo y el mantenimiento y la manipulación de la información (memoria de trabajo) cuando se emplean dígitos en orden inverso.

2.3.2.1 Letras y números (LN): consiste en la lectura, por parte del examinador, de una serie de números y letras desordenadas y el niño debe recordar la serie ordenando los números de menor a mayor y las letras por orden alfabético. La suma de ambos subtest permite obtener un índice compuesto de memoria de trabajo.

2.3.3 Test de clasificación de tarjetas de Wisconsin (WCST) (Heaton, Chelune, Talley, Kay \& Curtiss, 1997) Es una tarea que requiere habilidad para desarrollar y mantener las estrategias de solución de problemas que resultan adecuadas para conseguir un objetivo a través de condiciones que implican cambios de estímulos (Soprano, 2003). Específicamente brinda una medida del cambio o la flexibilidad cognitiva (Miyake et al., 2000). El test consiste en emparejar un mazo de dos bloques de 64 tarjetas respuesta, con 4 tarjetas estímulo (un triángulo rojo, dos estrellas verdes, tres cruces amarillas y cuatro círculos azules) que se colocan frente al sujeto. Cada vez que el sujeto empareja una tarjeta con una de las 4 tarjetas estímulo, se le indica si es correcto o incorrecto, pero nunca el criterio que debe tener en cuenta para clasificar. El test finaliza cuando se completan con éxito 6 categorías o hasta que se hayan empleado las 128 tarjetas. El indicador utilizado en el presente estudio como medida de la flexibilidad cognitiva fue el número de categorías completas.

2.3.4 Laberintos de Porteus (Porteus, 2006) Es una prueba que permite valorar la capacidad de planificación. Está constituido por doce laberintos de complejidad creciente, que según el nivel de dificultad, ofrece dos o cuatro intentos de resolución. El sujeto debe trazar con un lápiz el camino desde la entrada hasta la salida, sin repasarlo previamente con el dedo o el lápiz. Se puede aplicar en niños a partir de los 3 años de edad. El indicador empleado como medida de la planificación fue el número del total de laberintos completados correctamente.

\subsection{Procedimiento}

Una vez obtenido el consentimiento y asentimiento del niño se aplicaron los instrumentos de manera individual, en un periodo de tiempo de aproximadamente 40 minutos. En el caso de los niños que participaban de un taller de ajedrez, se le solicitó, además, un informe al profesor sobre el nivel de juego según su criterio y la cantidad de torneos en los que participó cada niño.

\subsection{Análisis de datos}

Para analizar las diferencias de rendimiento cognitivo según la práctica de ajedrez, se empleó Análisis Multivariado de Varianza (MANOVA). El procesamiento y análisis estadístico de los datos se realizó utilizando IBM SPSS Statistics versión 20.0. 


\section{RESULTADOS}

Los resultados indican que existen diferencias significativas en cuanto al rendimiento de tareas que valoran las FE según la práctica de ajedrez, $F$ de Hotelling $(4,60)=3,91, p=.007, \eta^{2}=.21$. Estas diferencias se encontraron para todas las FE evaluadas: inhibición $F(1,63)=7,00, p=.010, \eta^{2}=.10$, memoria de trabajo $F(1,63)=7,30, p=.009, \eta^{2}=.10$, flexibilidad cognitiva $F(1,63)=7,62, p=.008$, $\eta^{2}=.11$, y planificación $\mathrm{F}(1,63)=10,12, \mathrm{p}=.002, \eta^{2}=.14$, observándose puntuaciones medias superiores en los niños practicantes de ajedrez (ver Tabla 2).

Tabla 2.

Desempeño en tareas de FE según la práctica de ajedrez

\begin{tabular}{|c|c|c|c|c|c|c|}
\hline & \multirow{2}{*}{ Indicadores } & \multicolumn{2}{|c|}{ Practicantes de ajedrez } & \multicolumn{2}{|c|}{ No practicantes } & \multirow{2}{*}{$p$} \\
\hline & & M & $D E$ & $M$ & $D E$ & \\
\hline & Inhibición PC & 30.97 & 9.19 & 25.69 & 6.86 & .010 \\
\hline & IMT-WISC IV & 31.17 & 7.36 & 26.51 & 6.52 & .009 \\
\hline \multicolumn{7}{|l|}{ Funciones Ejecutivas } \\
\hline & FC-NCC & 3.70 & 2.02 & 2.49 & 1.52 & .008 \\
\hline & Plan-TL & 12.92 & 1.50 & 11.37 & 2.27 & .002 \\
\hline
\end{tabular}

\section{DISCUSIÓN}

El objetivo del presente estudio fue examinar las diferencias de rendimiento cognitivo en tareas que valoran las FE entre niños practicantes de ajedrez y no practicantes. Nuestros resultados indican que los niños que practican ajedrez presentan puntuaciones superiores en tareas que valoran la planificación, la inhibición, la memoria de trabajo y la flexibilidad cognitiva respecto de aquellos que no lo practican. Estudios previos también hallaron efectos del ajedrez, no solo en diferentes tareas que valoran las FE (Unterrainer et al., 2006; Rojas-Vidaurreta, 2001), sino también en dominios cognitivos como la habilidad meta-cognitiva y la resolución de problemas matemáticos (Kazemi et al., 2012), en el rendimiento académico en matemáticas y lengua (Gliga \& Flesner, 2014) y en el desarrollo socio-emocional de niños y adolescentes (Aciego et al., 2012). Específicamente en lo que refiere a la capacidad de planificación, se halló que los niños que practican ajedrez obtuvieron puntuaciones superiores respecto del grupo control.

Estas diferencias podrían explicarse por el hecho que, desde el inicio de cada partida, el sujeto debe planificar los movimientos a realizar, y anticiparse a las posibles jugadas de su oponente, para lograr 


\section{Larisa Ramos, Vanessa Arán Filippetti y Gabriela Krumm}

su objetivo. Específicamente, el jugador debe considerar todos los movimientos posibles, evaluar sus consecuencias y seleccionar el más apropiado. Si bien Cuéllar y Díaz (2009) no encontraron diferencias entre ajedrecistas profesionales, ajedrecistas aficionados y no practicantes en diferentes tareas que valoran la capacidad de planificación, autores como Unterrainer et al. (2006) y Aciego et al. (2012) sí hallaron diferencias en las tareas Torre de Londres y Laberintos de WISC-R, respectivamente, entre jugadores de ajedrez y no jugadores.

También se hallaron diferencias entre los grupos en cuanto a la capacidad de inhibición, siendo los niños practicantes de ajedrez los que demostraron tener un mejor control inhibitorio. Así, la inhibición sería una habilidad implicada en el ajedrez en tanto el sujeto debe ser capaz de prestar atención, seleccionar el movimiento más conveniente e inhibir los inapropiados y no apresurarse a efectuar una jugada sin considerar las posibles consecuencias.

A pesar de que las pocas investigaciones que relacionaron estas variables no han hallado diferencias significativas entre ajedrecistas y no practicantes (Nejati \& Nejati, 2012; Postal, 2012), es importante señalar que las mismas se han llevado a cabo con poblaciones de jóvenes y adultos expertos en ajedrez. Así, podría pensarse que la capacidad de inhibición sería importante durante la niñez y en los inicios de la práctica de ajedrez y la experticia explicaría, en parte, la mayor o menor demanda a los procesos inhibitorios durante la práctica de ajedrez.

Con respecto a la memoria de trabajo, los resultados también demostraron que sería una función implicada en las partidas de ajedrez, en tanto los niños que lo practican mostraron un rendimiento superior. Autores como Horgan y Morgan (1990), Schneider et al. (1993) y Robbins et al. (1996) también hallaron un efecto del ajedrez en diferentes tareas de memoria. Según Robbins et al. (1996) la memoria de trabajo, principalmente el componente ejecutivo central, estaría implicado en la selección de movimientos, es decir, en el proceso durante el cual se generan las secuencias de movimientos y se sopesan las ventajas de unos sobre otros (Torres \& Granados 2014). Finalmente, la flexibilidad cognitiva sería otra de las FE implicadas en el ajedrez. Nuestros resultados indican que los niños que practican ajedrez presentan puntuaciones superiores respecto de aquellos que no lo practican. Consistentemente, Rojas-Vidaurreta (2001) halló diferencias significativas en cuanto a la flexibilidad cognitiva entre niños ajedrecistas y un grupo control.

Según la autora, la flexibilidad cognitiva se pondría en juego cuando el sujeto debe plantear, organizar y reestructurar las jugadas considerando las reglas específicas del juego y las variaciones imprevistas del oponente. En síntesis, nuestros resultados indican que los niños que practican ajedrez muestran un mejor desempeño en tareas ejecutivas de planificación, inhibición, memoria de trabajo y flexibilidad cognitiva. Así, la práctica de ajedrez durante la niñez impondría demanda a diferentes procesos cognitivos ejecutivos, lo que sugiere que sería una herramienta efectiva para favorecer el desarrollo de las FE durante la niñez. Según Remine, Care y Brown (2008) la habilidad para anticipar, planificar los movimientos antes de realizarlos y analizar la efectividad de los movimientos ejecutados serían los procesos ejecutivos implicados en el juego de ajedrez.

Los resultados de este estudio tienen importantes implicaciones para el ámbito clínico, educativo y familiar. En el ámbito clínico, estos datos tienen implicancia para la intervención terapéutica con 


\section{Larisa Ramos, Vanessa Arán Filippetti y Gabriela Krumm}

poblaciones infantiles, particularmente con aquellas que presenten un patrón neurocognitivo de disfunción ejecutiva. Al respecto, un estudio reciente demostró una mejoría en los síntomas de desatención e hiperactividad en niños con TDAH tras la aplicación de un programa de entrenamiento ajedrecista (Blasco-Fontecilla et al., 2016). Así, podría pensarse al ajedrez como una estrategia terapéutica útil para la estimulación de las FE en la clínica infantil. Por otra parte, los resultados del presente estudio tienen implicancias para el ámbito educativo en tanto ponen de manifiesto la importancia del ajedrez para el desarrollo de FE necesarias para el aprendizaje escolar. Esto promueve el diseño de estrategias de entrenamiento en ajedrez para favorecer el desarrollo de las $\mathrm{FE}$, y del rendimiento académico en general, desde el inicio de la escolaridad formal. Según RojasVidaurreta (2001), la práctica de ajedrez favorece el desarrollo ejecutivo de las personas, lo que a su vez potencia la resolución de problemas en la vida diaria.

Finalmente, estos datos tienen implicancias para el ámbito familiar en tanto revelan la posibilidad de estimular lúdicamente las FE a través de la práctica de ajedrez. Para la realización de futuras investigaciones sería relevante estudiar el efecto de la práctica de ajedrez en las FE en poblaciones adolescentes y adultas, para comprobar si la experticia y la edad podrían explicar, en parte, las variaciones individuales de funcionamiento ejecutivo según la práctica de ajedrez. Por otra parte, consideramos importante evaluar las FE en instituciones educativas en donde se utilice el ajedrez como herramienta pedagógica de apoyo para el rendimiento académico.

\section{REFERENCIAS}

Aciego, R., García, L., \& Betancort, M. (2012). The benefits of chess for the intellectual and social-emotional enrichment in schoolchildren. The Spanish Journal of Psychology, 15(2), 551-559. https://doi.org/10.5209/rev_SJOP.2012.v15.n2.38866

Arán-Filippetti, V. (2011). Funciones ejecutivas en niños escolarizados: efectos de la edad y del estrato socioeconómico. Avances en psicologia Latinoamericana, 29(1), 98-113. Disponible en http://revistas.urosario.edu.co/index.php/apl/article/view/499

Arán-Filippetti, V. (2013). Structure and invariance of executive functioning tasks across socioeconomic status: evidence from spanish-speaking children. The Spanish Journal of Psychology, 16(e101), 1-15. DOI: http://dx.doi.org/10.1017/sjp.2013.102

Arnau, J. (1978). Métodos de investigación de las ciencias Humanas. Barcelona: Omega.

Baddeley, A.D. (1992). Working memory. Science, 255(5044), 556-559. DOI: 10.1126/science.1736359

Blasco-Fontecilla, H., González-Pérez, M., García-López, R., Poza-Cano, B., Pérez-Moreno, M. R., de León Martínez, V., \& Otero-Pérez, J. (2016). Eficacia del ajedrez en el tratamiento del trastorno por déficit de atención e hiperactividad: un estudio prospectivo abierto. Revista de Psiquiatría y Salud Mental, 9(1), 13-21. DOI: 10.1016/j.rpsm.2015.02.003

Calle, D. (2017). Filogenia y desarrollo de funciones ejecutivas. Psicogente, 20(38), 368-381. http://doi.org/10.17081/psico.20.38.2557 
Larisa Ramos, Vanessa Arán Filippetti y Gabriela Krumm

Collette, F., Hogge, M., Salmon, E., \& Van der Linden, M. (2006). Exploration of the neural substrates of executive functioning by functional neuroimaging. Neuroscience, 139(1), 209-221. DOI: 10.1016/j.neuroscience.2005.05.035

Cortada de Kohan, N., Macbeth, G., \& López-Alonso, A. (2008). Técnicas de investigación científica. Buenos Aires: Lugar Editorial.

Cuéllar, J., \& Díaz, A. (2009). Desempeño en pruebas de funciones ejecutivas que miden el componente de planificación en un grupo de 30 ajedrecistas profesionales, aficionados y no practicantes de este deporte en la ciudad de Bogotá. (Tesis de grado inédita). Pontificia Univesidad Javeriana, Colombia. Disponible en https://repository.javeriana.edu.co/handle/10554/7995

Dauvergne, P. (2000). The case for chess as a tool to develop our children's minds. Recuperado de http://www.auschess.org.au/articles/chessmind.htm

Diamond, A. (2016). Why improving and assessing executive functions early in life is critical. Executive function in preschool age children: Integrating measurement, neurodevelopment and translational research. Washington, DC: American Psychological Association.

Fernández-Amigo, J., \& Sánchez-Rincón, M. (2011). Canto al ajedrez: enseñar ajedrez con canciones. Tendencias pedagógicas, 18(2011), 269-322. DOI: 10.15366/tp

Ferreira, D., \& Palhares, P. (2008). Chess and problem solving involving patterns. The Montana Mathematics Enthusiast, 5, 249-256. Disponible en https://scholarworks.umt.edu/cgi/viewcontent.cgi?article=1105\&context=tme

Fraile-Aranda, A. (2004). El deporte escolar en el siglo XXI: análisis y debate desde una perspectiva europea. Barcelona: Graó.

Gliga, F., \& Flesner, P. I. (2014). Cognitive benefits of chess training in novice children. Procedia-Social and Behavioral Sciences, 116(21), 962-967. https://doi.org/10.1016/j.sbspro.2014.01.328

Gobet, F., \& Campitelli, G. (2002). Intelligence and chess. In J. Retschitzki, \& R. Haddad-Zubel, (Eds.). Step by step. Proceedings of the 4th Colloquium "Board Games in Academia". (pp. 103-112). Fribourg: Editions Universitaires.

Gobet, F., \& Campitelli, G. (2006). Educational benefits of chess instruction: A critical review. In Chess and education: Selected essays from the Koltanowski conference (pp. 124-143). Dallas: Programa de Ajedrez de la Universidad de Texas.

Golden, C. J. (2007). Stroop, Test de Colores y Palabras. Madrid: TEA Ediciones.

Heaton, R. K., Chelune, G. J., Talley, J. L., Kay, G. G., \& Curtiss, G. (1997). WCST, Test de clasificación de tarjetas de Wisconsin. Madrid: TEA Ediciones.

Hong, S. \& Bart, W. M. (2007). Cognitive Effects of Chess Instruction on Students at Risk for Academic Failure. International Journal of Special Education, 22(3), 89-96. Disponible en https://eric.ed.gov/?id=EJ814515

Horgan, D. D., \& Morgan, D. (1990). Chess expertise in children. Applied cognitive psychology, 4(2), 109-128. DOI: 10.1002/acp. 2350040204 


\section{Larisa Ramos, Vanessa Arán Filippetti y Gabriela Krumm}

Kazemi, F., Yektayar, M., \& Abad, A. M. B. (2012). Investigation the impact of chess play on developing meta-cognitive ability and math problemsolving power of students at different levels of education. Procedia-Social and Behavioral Sciences, 32, 372-379. https://doi.org/10.1016/j.sbspro.2012.01.056

Kovacic, D. M. (2012). Ajedrez en las escuelas: una buena movida. PSIENCIA: Revista Latinoamericana de Ciencia Psicológica, 4(1), 29-41. http://dx.doi.org/10.5872/psiencia.v4i1.87

Lehto, J. E., Juujärvi, P., Kooistra, L., \& Pulkkinen, L. (2003). Dimensions of executive functioning: Evidence from children. British Journal of Developmental Psychology, 21(1), 59-80. http://dx.doi.org/10.1348/026151003321164627

Miyake, A., Friedman, N. P., Emerson, M. J., Witzki, A. H., Howerter, A. \& Wager, T. D. (2000). The unity and diversity of executive functions and their contributions to complex "Frontal Lobe" tasks: a latent variable analysis. Cognitive Psychology, 34 41(1), 49-100. http://dx.doi.org/10.1006/cogp.1999.0734

Montero, I. \& León, O. G. (2007). A guide for naming research studies in Psychology. International Journal of clinical and Health psychology, 7(3), 847-862. Disponible en http://www.aepc.es/ijchp/GNEIP07 es.pdf

Nejati, M. \& Nejati, V. (2012). Frontal lobe function in chess players. Acta Medica Iranica, 50(5), 311-314. Disponible en https://www.ncbi.nlm.nih.gov/pubmed/22837083

Porteus, S. D. (2006). Laberintos de Porteus (4ㅁ edición revisada). Madrid: TEA Ediciones.

Postal, V. (2012). Inhibition of irrelevant information is not necessary to performance of expert chess players. Perceptual \& Motor Skills, 115(1), 60-68. https://doi.org/10.2466/23.04.22.PMS.115.4.60-68

Remine, M. D., Care, E. \& Brown, P. M. (2008). Language ability and verbal and nonverbal executive functioning in deaf students communicating in spoken English. Journal of deaf studies and deaf education, 13(4), 531-545. https://doi.org/10.1093/deafed/enn010

Robbins, T. W., Anderson, E. J., Barker, D. R., Bradley, A. C., Fearnyhough, C., Henson, R. \& Baddeley, A. D. (1996). Working memory in chess. Memory \& Cognition, 24(1), 83-93. DOI: 10.3758/ BF03197274

Rojas-Vidaurreta, L. (2001). Aproximación al estudio de la flexibilidad cognitiva en niños ajedrecistas. Revista Cubana de Medicina Deportiva y Cultura Física, 6(2). https://doi.org/10.1080/02109395.2017.1295578

Schneider, W., Gruber, H., Gold, A., \& Opwis, K. (1993). Chess expertise and memory for chess positions in children and adults. Journal of Experimental Child Psychology, 56(3), 328-349. https://doi.org/10.1006/jecp.1993.1038

Soprano, A. M. (2003). Evaluación de las funciones ejecutivas en el niño. Revista de Neurología, 37(1), 44-50. Disponible en

http://www.neurodesarrollo.net/cursos/images/Descarga/Neurodesarrollo/Unidad\%206/SopranoFE_Ni\%C3 \%B1o.pdf

Spiendler, S., Carlotto, M., Ogliari, D., Giordani, K. (2015). Estressores ocupacionais em psicólogos clínicos brasileiros. Psicogente, 18(33), 104-116. http://doi.org/10.17081/psico.18.33.59

Tamorri, S. (2004). Neurociencias y deporte. Psicología deportiva, procesos mentales del atleta. Barcelona: Paidotribo

Thompson, M. (2003). Does the playing of chess lead to improved scholastic achievement? Issues in Educational Research, 13, 13-26. Disponible en https://saintlouischessclub.org/education/research/does-playing-chesslead-improved-scholastic-achievement 


\section{Larisa Ramos, Vanessa Arán Filippetti y Gabriela Krumm}

Tirapu-Ustárroz, J., Muñoz-Céspedes, J. M., Pelegrín-Valero, C., \& Albéniz-Ferreras, A. (2005). Propuesta de un protocolo para la evaluación de las funciones ejecutivas. Revista de neurología, 41(3), 177-186. Dsiponible en https://www.neurologia.com/articulo/2005054

Torres, P. \& Granados, D. E. (2014). Procesos cognoscitivos implicados en la comprensión lectora en tercer grado de educación primaria. Psicogente, 17(32), 452-459.

Unterrainer, J. M., Kaller, C. P., Halsband, U., \& Rahm, B. (2006). Planning abilities and chess: A comparison of chess and non-chess players on the Tower of London task. British Journal of Psychology, 97(3), 299-311. DOI: $10.1348 / 000712605 \times 71407$

Waters, A. J., Gobet, F., \& Leyden, G. (2002). Visuospatial abilities of chess players. British Journal of Psychology, 93(4), 557-565. DOI: 10.1348/000712602761381402

Wechsler, D. (2010). WISC IV, Escala de Inteligencia para niños de Wechsler - IV. Adaptación Argentina. Buenos Aires: Paidós.

Está obra está bajo: Creative commons attribution 4.0 international license. El beneficiario de la licencia tiene el derecho de copiar, distribuir, exhibir y representar la obra y hacer obras derivadas siempre y cuando reconozca y cite la obra de la forma especificada por el autor o el licenciante 